\title{
Auditory autokinesis: Effects of signal frequency and intensity*
}

\author{
JAMES L. FOBES $\uparrow$, University of Arizona, Tucson, Ariz. 85721
} and

DAVID R. PERROTT, California State College, Los Angeles, Calif. 90032

An experiment is reported on auditory autokinesis performed under diotic listening conditions. Tests were conducted with pure tones of $500,1,000,2,000$, and $5,000 \mathrm{~Hz}$ at signal levels of $25,40,55,70$, and $85 \mathrm{~dB}$ sound pressure level. Neither signal frequency nor signal intensity level, over the range of values tested, had any significant systematic effect on the obtained function. These results do not support a localization-precision or a sensory-adaptation hypothesis, which have been proposed to account for auditory autokinesis.

Since the initial report, by Bernardin \& Gruber (1957), of a true auditory autokinetic effect (as opposed to apparent and actual source displacement, or "real" movement), there has been confusion about the parameters of signal intensity and frequency. Bernardin and Gruber used three pure tones $(56,560$, and $5,600 \mathrm{~Hz}$ ) presented at an unspecified intensity. Some indication of a possible frequency effect was obtained, but without quantitative support (e.g., listeners reported that the higher pitch tone appeared to move more dramatically). This indication of a frequency effect in auditory autokinesis was later contradicted by Anderson \& Moss (1964), who used a combination of four frequencies $(1,000,2,000,5,000$, and $10,000 \mathrm{~Hz}$ ) and three intensity levels $(20 \%, 40 \%$, or $60 \%$ of the output of a Heathkit audio oscillator). This investigation reported the absence of an effect due to either parameter. In the following year (1965), Cautela \& McLaughlin presented evidence for an intensity effect obtained with two signal levels (35 and $70 \mathrm{~dB}$ ); more movement responses were given with the more intense stimulus. These studies appear to be preliminary. In general, little attention was paid to the specification of the stimulus parameters of the test situation. Reports utilized qualitative descriptions with relatively little quantification of the data.

\section{AUDITORY AUTOKINESIS}

UNDER DIOTIC LISTENING CONDITIONS

In these earlier studies, the use of an open-field procedure (signal presented via loudspeaker), under nonanechoic

*This research was supported in part by Associated Students of Califomia State College Grant ACCT 4585 and by Training Grant MH 11286 from the U.S. Public Health Service to the senior author.

tR equests for reprints should be directed to: J. L. Fobes, Department of Psy chology. University of Arizona, Tueson, Ariz. 85721. conditions, suggested that the autokinetic effect could be an acoustic artifact produced by standing waves in the test chamber. Any change in the standing wave pattern could produce actual changes in interaural stimulation (as would be generated if the source were actually moved). To test this possibility, Perrott \& French (1969) used both an open-field (again nonanechoic) and a diotic listening procedure. The results of this study indicated that apparent localization shifts could be obtained in both the free-field (loudspeaker) and diotic (stereophones) test situations; the notion that auditory autokinesis was a simple acoustic artifact produced by nonanechoid conditions was not supported. However, in this experiment, a signal effect was observed. Both a $1,000-\mathrm{Hz}$ tone and white noise were used as stimuli; considerably more movement was observed with the tonal stimulus than with the white noise.

Perrott \& Fobes (1971) performed a study to investigate this effect more systematically. In this study, experimental conditions were defined by the bandwidth of the test signal (pure tone, one-octave, two-octave, and wide-band noise). Each signal had a center frequency of approximately $1,000 \mathrm{~Hz}$. The results of this study confirmed the earlier observations. Both the duration and the number of autokinetic responses were inversely related to signal bandwidth. In addition, latency to the first response appeared to be directly related to bandwidth, but, due to large variance within groups, this effect was not significant.

The evidence collected thus far on an auditory autokinetic effect indicates that: (1) auditory autokinesis can be observed in a number of different situations, even without specific movement instructions (Bernardin \& Gruber, 1957), but more reports of movement are obtained when specific reference to moving sounds is given (Cautela \& McLaughlin, 1965); (2) signal frequency may not be a significant factor in auditory a utokinesis (Anderson \& Moss, 1964), although Bernardin \& Gruber (1957) indicated that the magnitude of movement appeared to be greater with high-frequency sounds; and (3) signal intensity may be a significant factor (Cautela \& McLaughlin, 1965), although others have not observed this effect (Anderson \& Moss, 1964).

Auditory autokinesis does appear to be related to the bandwidth of the acoustic stimulus; this bandwidth effect is suggestive. In open-field listening, for example, listeners can localize a source generating clicks and wideband noise more accurately than they can pure tones (Stevens \& Newman, 1936; Howard \& Templeton, 1966). Autokinesis then may be inversely related to the conditions necessary for localization precision. In numerous studies (Stevens \& Newman, 1936; Mills, 1958; Perrott, 1969), investigators have observed that localization precision, in open-field conditions, is good for both low- and high-frequency stimuli but relatively poor for signals of intermediate frequencies $(1,500$ to $3,000 \mathrm{~Hz})$. Signal intensity level also appears to be systematically related to spatial localization precision, with the precision of lateralization improving as signal level is raised from low (15-dB sensation level) to intermediate (70- $\mathrm{dB}$ sensation level) signals levels (Elfner \& Perrott, 1967).

If auditory autokinesis is related to localization precision, then the autokinetic function should vary as a function of stimulus frequency and intensity. Bernardin \& Gruber (1957) used signals at 56,560 , and $5,600 \mathrm{~Hz}$. Considering the above discussion, this was a rather unfortunate choice of stimuli. Anderson \& Moss (1964) report having examined frequencies of $1,000,2,000,5,000$, and $10,000 \mathrm{~Hz}$, but their procedure involved short stimulus periods $(30 \mathrm{sec})$. In view of the long latencies observed by Perrott \& Fobes (1971), for the first autokinetic response (most falling within a range of 40 to $100 \mathrm{sec}$ ), this $30-\mathrm{sec}$ test period may have been too limited.

Tests conducted under diotic conditions would provide the best stimulus control. Some additional characteristics, unique to the diotic task, must be considered. The limits for the utilization of interaural phase information is essentially the same for both earphone and free-field tasks (i.e., an upper-frequency limit somewhere between 1,000 and $1,500 \mathrm{~Hz}$ exists for both), but interaural effects for signal intensity in 
the free field are determined by headshadow, a function of little relevance to the diotic listener. However, the utilization of interaural intensity differences is a function of frequency, even under earphone presentation, although this limit appears to be set by the auditory system itself (Mills, 1960; Rowell, 1970). The system is relatively insensitive to interaural intensity differences at $1,000 \mathrm{~Hz}$ but becomes more sensitive to both lower and higher frequencies (optimal low frequencies, 100 to $500 \mathrm{~Hz}$ ), with a slight advantage to the higher frequencies.

The following study examined the role of both signal intensity and signal frequency on the diotic autokinetic effect.

\section{SUBJECTS}

One hundred and twenty Ss were employed. Each $S$ was selected on the basis that hearing in both ears was within normal limits.

\section{APPARATUS}

Sine-wave signals were led from an oscillator (General Radio, Model 1311A) through a transformer, where the signal was split. Two signals were then led from the splitter to separate precision attenuators, switches, and impedance-matching transformers. Each signal was led to one side of a pair of earphones (Grason-Stadler Stereophones, Model TDH-49). Initial calibrations of signal amplitude and frequency were made at the earphones using a 6-cc coupler and sound-level meter (General Radio, Model 5151C). On-line checks of signal frequency and amplitude were made regularly with an electronic counter, rms voltmeter, and oscilloscope. The signals at both sides of the earphones were matched for phase, frequency, and amplitude.

\section{PROCEDURE}

The auditory autokinetic tests were conducted under diotic listening conditions. Ss were tested individually in an audiometric test room (Industrial Acoustics Co., Model 1200). Ss were instructed to press a response key if the auditory tonal "image" appeared to move and to hold the key down for the entire period of movement, if any. A keypress activated a timer that recorded duration of movement, and a counter indicated the total number of responses. Ss were further instructed that they would not necessarily hear movement.
Four frequencies were used $(500$, $1,000,2,000$, and $5,000 \mathrm{~Hz}$ ) in combination with five intensity levels $(25,40,55,70$, and $85 \mathrm{~dB})$ ( sound-pressure level, re: 0.0002 dynes $/ \mathrm{cm}^{2}$ ). Ss were assigned randomly to be tested for $10 \mathrm{~min}$ with one frequency at one intensity level. Within each of these 20 stimulus combinations (four frequencies with five levels of each), six Ss were tested.

\section{RESULTS}

The results of the study indicate that neither signal frequency nor signal amplitude have a significant systematic effect on auditory autokinesis. A separate 4 by 5 analysis of variance was performed on both the number and duration of autokinetic responses as a function of signal frequency and amplitude. No significant differences were observed between the four frequencies and five intensity levels employed for either dependent variable. No significant interactions were observed between signal frequency and signal amplitude.

Of the 120 Ss observed, 26 reported no autokinesis (21.6\%), 7 made only 1 response $(5.8 \%)$, and 8 Ss $(6.6 \%)$ responded at a rate in excess of $3 / \mathrm{min}$. Most Ss reported between 5 and 20 autokinetic events in the 10-min session ( $66.0 \%$ of the Ss). The mean response rate, 1.02 responses $/ \mathrm{min}$, computed across treatments, appears to be representative of the behavior of most Ss. Similarly, the mean duration of an auditory autokinetic response to the tonal stimuli employed in this study, $8.2 \mathrm{sec}$, also appears to be representative.

\section{DISCUSSION}

The results of this study clearly do not support the notion that either frequency or intensity of the signal affects auditory autokinesis. This finding is in agreement with the earlier report by Anderson \& Moss (1964). Insofar as localization precision is related to both signal frequency and intensity, the present results do not seem to indicate any relationship between localization precision and auditory autokinesis.

An alternative explanation for auditory autokinesis, which rests on sensory adaptation effects, has been offered by Perrott \& Fobes (1971). Adaption to tonal stimuli, however, is also a function of both the frequency and level of the signal. For example, Caussé \& Chavasse (1947) reported that for stimuli between 300 and
$1,000 \mathrm{~Hz}$, the rate of adaptation, defined in terms of temporary threshold shift, increased as a function of an increase in frequency, reaching a maximum at $1,000 \mathrm{~Hz}$. Between 1,000 and $9,000 \mathrm{~Hz}$, the function approximates a constant. Rate of adaptation was directly related to signal level. The present results clearly do not provide support for this hypothesis either.

Inspection of general performance with tonal stimuli presented in the present study and data collected in earlier diotic work indicates a surprising degree of similarity. Perrott \& French (1969) reported that with a $1,000-\mathrm{Hz}$ tone, the mean duration of an autokinetic response was $5.1 \mathrm{sec}$. In the Perrott \& Fobes (1971) study, the mean response duration (again with a $1,000-\mathrm{Hz}$ test tone) was $9.8 \mathrm{sec}$. In the present study, the mean duration was $8.2 \mathrm{sec}$.

\section{REFERENCES}

ANDERSON, D. C., \& MOSS, C. A. The auditory autokinetic effect. American Joumal of Psychology, 1964, 77,502.

BERNADIN, A. C., \& GRUBER, H. E. An auditory autokinetic effect. American Journal of Psychology, 1957, 70, 133.

CAUSSÉ, R., \& CHAVASSE, P. Etudes sur la fatigue auditive. Année Psychologie, $1947,43-44,265-298$.

CAUTELA, J. R., \& MCLAUGHLIN, D. The influence of suggestion on the audiokinetic effect. Journal of Psychology, 1965, 60, 117.

ELFNER, L. F \& PERROTT, D. $R$. Lateralization and intensity discrimination. Joumal of the Acoustical Society of America, 1967, 41, 441 .

HOWARD, I. P., \& TEMPLETON, W. B. Human spatial orientation. London: Wiley, 1966.

MILLS, A. W. On the minimum audible angle. Joumal of the Acoustical Society of America, $1958,30,237$.

MILLS, A. W. Lateralization of high frequency tones. Journal of the Acoustical Society of America, 1960, 32. 132.

PERROTT, D. R. Role of signal onset in sound localization. Journal of the Acoustical Society of America, 1969, 45, 436.

PERROTT, D. R., \& FRENCH, D. J. Apparent movement of sounds: Auditory autokinesis. Psychonomic Science, 1969. $17,207$.

PERROTT, D. R., \& FOBES, J. L. Autokinesis as a binaural localization phenomenon: Effects of signal bandwidth. Jourmal of Experimental Psychology, 1971,87, 172.

ROWELL, D. Auditory display of spatial information. Unpublished dissertation, University of Canterbury, Christchurch, New Zealand, 1970.

STEVENS. S. S.. \& NEWM AN, E. B. The localization of actual sources of sound. American Journal of Psychology, 1936, 48, 297. 